\title{
DEVELOPMENT OF A TEACHING MATERIAL SYSTEM FOR THE FUNDAMENTAL MATHEMATICS EDUCATION FOR INFORMATION, COMPUTERS AND SYSTEMS ENGINEERING
}

\author{
Masami Iwase, Shoshiro Hatakeyama, Katsuhisa Furuta \\ Department of Computers and Systems Engineering, Tokyo Denki University \\ Hatoyama, Hiki-gun, Saitama, JAPAN, 350-0394 \\ Tel: +81-49-296-2911 / Fax: +81-49-296-6185 \\ E-mail: iwase@k.dendai.ac.jp / URL: http://furutalab.k.dendai.ac.jp
}

\begin{abstract}
Recently, in especially private college in Japan, dispersion of students' educational achievement is distinguished, and fundamental educations for freshmen become more important. The education materials, which raise the level of low-achievement students and interest other students simultaneously, are required. Then in this paper, we present a teaching material system and project-style education of fundamental mathematics for freshmen, which was demonstrated in our department. It is highlighted that using this system we assign each student each data composing a project, so that we succeed in not only improving education effect but also constructing the decentralized data-making/acquisition system with students.
\end{abstract}

Keywords: - distributed-education system, project-type education, decentralized data-making/acquisition system

\section{INTRODUCTION}

In this paper, we present a teaching material system and project-style education of fundamental mathematics for freshmen, which was demonstrated in our department. Recently, in especially private college in Japan, the entrance examinations are diversified, and it succeeds in collecting various type students. On the other hand, we face the problem that fundamental achievement of freshmen varies from high to low as a side effect of diversified examination. We find empirically that the cause of the problem is not student's original ability but is that students have not learned enough before they enter the college. Therefore, if students had low achievement, they can recover it by taking the appropriate education in college.

In the point of lecturer's view, we have to give the same lecture to all students simultaneously even if students' achievement levels are different obviously. One of the education strategies is to plan an improvement of the low-level achievement students, but this strategy is afraid of chilling the interests of the average students. In opposite case, the low-level achievement student probably cannot follow the progress of the lecture. The important point is to choose contents of the lecture, which can be understood by all students and can interest the average or more high-achieved students.

In the case of our department reported in this paper, we adopt the complex value, which is required as basic notion for information and control system, as a theme of the lecture. The notion of complex value is usually learned in high school of Japan, but even if a student would not learn the complex value, he could enter to our department. Then, we taught the complex value as a target for the students. Because almost students have known the complex value, the lecture might become dull if lecturer taught in the conventional way. However almost students have not learned fractals yet, we used fractals such as Julia and Mandelbrot sets as teaching materials to interest them. Prof. Mandelbrot also said that the fractal is a very good material for fundamental mathematics education [1]. Furthermore, via fractals, we expect that students can learn not only complex value but also the Euler's formula, coordinate transformation, dynamical system and stability of the system, which are important notions for our department. In this paper, we present a project-style lecture and a teaching material system developed to support the lecture, discuss it, and show its effectiveness by giving the actually practiced lecture in our department as an example. 


\section{GRAPHICS SYSTEM FOR MATX}

\subsection{EDUCATION ENVIRONMENT}

The education environment is an important factor to realize the proposed-style lecture. In our style, it is required for each student to have a personal computer in order to draw computer graphics. Fortunately we strongly recommend and instruct all students in this department to have a laptop PC, and we could make the most of this situation. Moreover, in our campus, both network ports and wire-less network access points are equipped, and all students can access and utilize the internet whenever and wherever they need it. These education environments play a role of helping and motivating staffs ourselves to consider the better education ways, means and resources.

\subsection{MATX}

In information and system engineering, we often face the situations that various numerical algorithms and calculations are required to solve the problems. Therefore useful tools such as MATRIXx [2] and MATLAB [3] are developed and used frequently. A freeware called "MaTX" developed by Prof. M. Koga [4] is an excellent programming language with good descriptiveness like $\mathrm{C}$, and equips matrices calculation, numerical algorithm for solving the ordinary differential equation, and others needed for engineering. [5][6] Since MaTX equips the famous matrices calculation algorithm, Linpack [7] and Eispack, [8] then it has almost same functions of basic ones in MATLAB. Moreover, MaTX can run on Linux Solaris, FreeBSD, Windows, DOS and many other OS. (See the detail in http://www.matx.org.) We could receive enough benefit of MaTX, but on the other hand, to our regret, we were also dissatisfied with MaTX's graphics and audio abilities. This is one of the motivations to develop a graphics system for MaTX introduced in the next section.

\subsection{MXGRAPHICS}

Considering about the education in information and system engineering, we think that results of some operations done by students should be visible for them to feedback whether the operation is correct or not. Using MaTX, we obtained the numerical calculation tools, but did not obtain enough representation way. Therefore we made a teaching material system, which we called "MXGraphics", for beginners to draw computer graphics such as fractals easily with MaTX. The MXGraphics is made with simple structure. This consists of the thread for command processing and communication with MaTX, the thread for drawing, and a cue-buffer connecting two threads as shown in Fig. 1.

Since almost all numerical calculation to draw the computer graphics can be done by MaTX, users

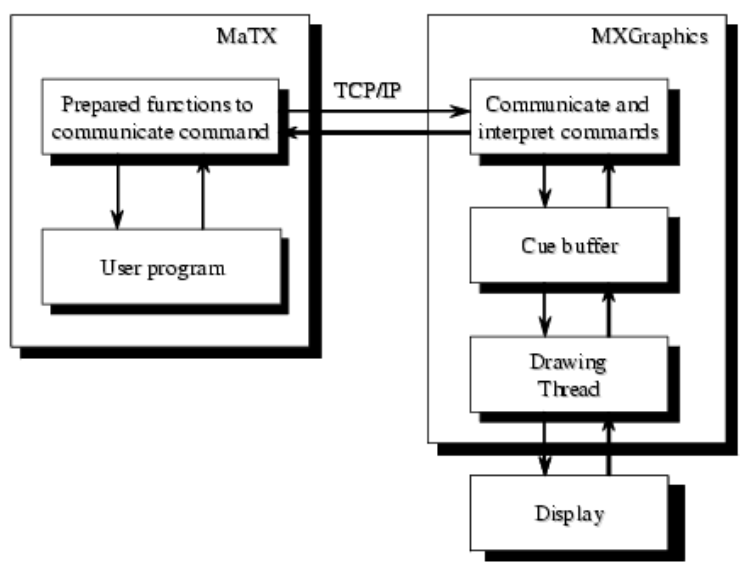

Fig.1 - Structure of MXGraphics system with MaTX.

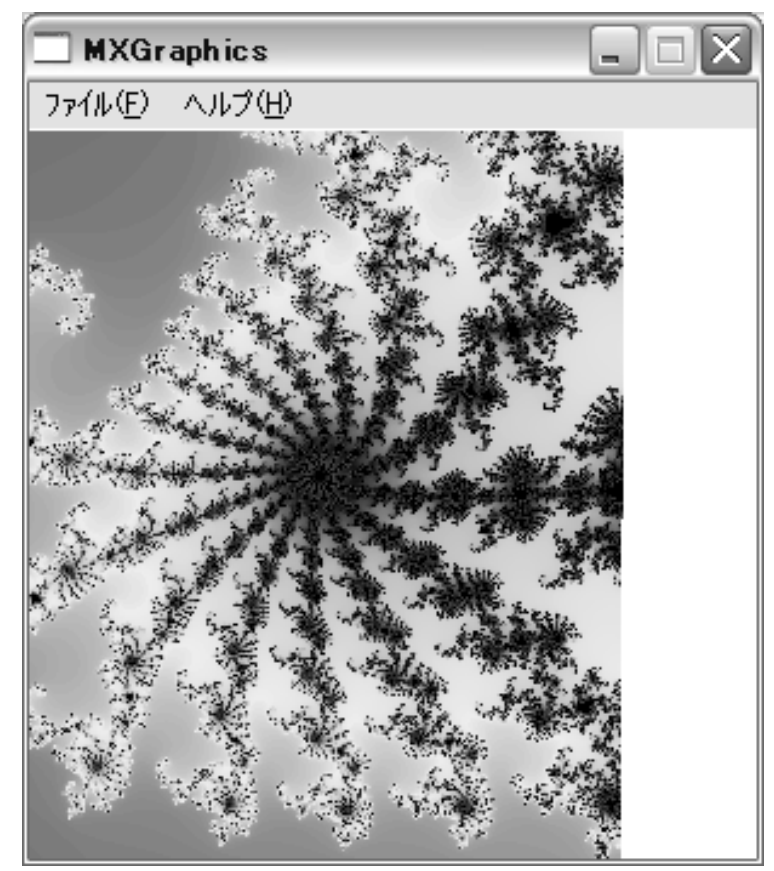

Fig.2 - MXGraphics window running on Window®. should only send commands to the MXGraphics by

Table 1 - List of MXGraphics commands

\begin{tabular}{|c|c|}
\hline Commands & Action \\
\hline MXG_open() & Open a MXGraphics window \\
\hline MXG_close () & Close a MXGraphics window \\
\hline MXG_clear() & Clear a canvas \\
\hline MXG_bg() & Set the background color \\
\hline MXG_point() & Dot a point \\
\hline MXG_line() & Draw a line \\
\hline MXG_rect() & Draw a rectangle \\
\hline MXG_ellipse() & Draw a ellipse \\
\hline
\end{tabular}

using some functions prepared. Then the MXGrpahics receives the drawing-commands via TCP/IP network, and interprets the commands and draws. Fig. 2 is a picture of the MXGraphics window drawing a fractal. A drawn computer 
graphic by MXGraphics can be saved into a windows bitmap file by clicking the menu of $[\operatorname{File}(\mathrm{F})]$ and that of [Save as a new file (A)]. The drawing commands used in the user MaTX program are shown in Table 1.

\section{PROJECT STYLE LECTURE ON FUNDAMENTAL MATHEMATICS}

We planned a lecture on fundamental mathematics using fractal as a materials, which can be understood by the low-level achievement students and motivate average students simultaneously. To support this lecture, we developed a distributed teaching system, MXGraphics, utilizing the MaTX. Giving these tools students, they have the environment to draw computer graphics easily. Now we will present how we utilized the educational environment effectively, and practiced a project-style lecture in our department actually.

\subsection{MAKING A FRACTAL MOVIE AS A PART THE PROJECT-STYLE LECTURE}

In the conventional lecture style, we fear that a lecturer gives kind of one-sided lecture. We present a project style lecture in order to prompt students to join the lecture actively. In the presented style, a lecturer plans the lecture schedule to complete a project as a whole, assigns a part of the project to each student, and gives them responsibility for the jobs. We can expect students to have clear purpose of joining in the lecture, and avoid dropping out the lecture.

For example our project is shown here. The project is not complex but very simple. We planned making a fractal movie all together teaching a complex value, Euler's formula, introduction of dynamical system and so on through drawing fractals such as the Julia and Mandelbrot set. The project consists of twelve lectures a half year. The contents of each lecture are important for information and system engineering, and also are required to make the fractal movie. We made an effort at planning the schedule in order to make students equip the required knowledge and skill naturally through the project, and in order to make students sense that learning the contents in each lecture is required to achieve the project. The lecture scheduled is as following.

Lecture schedule:

1. Introduction (Explain the purpose and scope)

2. Computer literacy (operation of Windows and tutorial of MaTX and MXGraphics)

3. Complex number, Gauss-plane, Polar-coordinate

4. Series, radius of convergence and exponential function

5. Euler's formula in complex analysis

6. Complex calculation and coordinate transformation

7. Introduction of dynamical system and stability

\section{Intermediate}

9. Julia set (monochrome version)

10. Julia set (color version) and assignment of each

fractal graphics to make a fractal movie

11. Mandelbrot set

12. Finals

The concrete contents of the project is to make a fractal movie consisting of many still computer graphics of Julia set drawn with different parameter respectively.

In general, the Julia set is drawn according to the following equation:

$$
z[k+1]=z^{2}[k]+C
$$

With a given complex-value parameter $C$ and an initial value $z[0]$ in the Gauss-plane, $z[k]$ can be obtained calculating Eq. (1) repeatedly. Then, we make a color correspond to the period in which $z[k]$ keeps remaining in a region, that is, we assign a color according to when the state $z[k]$ gets out from the considered region. (See Fig. 3.) Then, we dot at the initial value $z[0]$ in the Gauss-plane with the decided color. Repeating the above procedure for all dots in the drawing area, the computer graphics of the Julia set can be obtained. However, if anyone uses Eq. (1) to draw the Julia set, of course, everyone gets the same results.

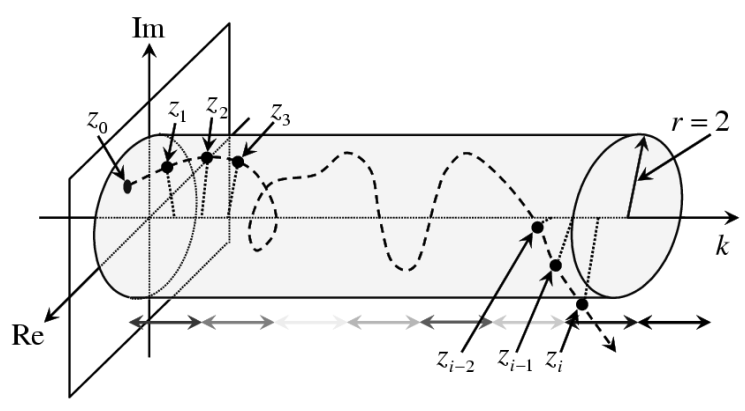

Fig.3 - How to draw the Julia set.

Therefore, we introduced a parameter $\alpha$ into the difference equation (1) as follows:

$$
z[k+1]=z^{\alpha}[k]+C
$$

and assigned the different parameter $\alpha$ from 1.9 to 2.01 to each student individually. The students made the fractal graphics with the given parameters, and saved the graphics to windows bitmap files. In Fig. 4, some examples of the fractal graphics made by the students are shown. These files were collected to a server via FTP, and were linked to make a fractal movie. This is the all of the project. Moreover this style of lecture has other good features, i.e. students can easily find the results of their contribution of the project. If a student makes a wrong graphics, then it can be found immediately that a movie including the graphics might be strange, because the frames of fractal movie should change 
continuously. To complete the movie, the student will revise the applicable graphics. We can find that a feedback process to the students works in the project-style lecture.

\subsection{DECENTRALIZED DATA MAKING AND ACQUISITION SYSTEM}

In the aforementioned section, we presented the education material system and the project-style lecture. In addition, we discuss the lecture style from another point of view. Let us consider the total time needed to make a fractal movie. In our
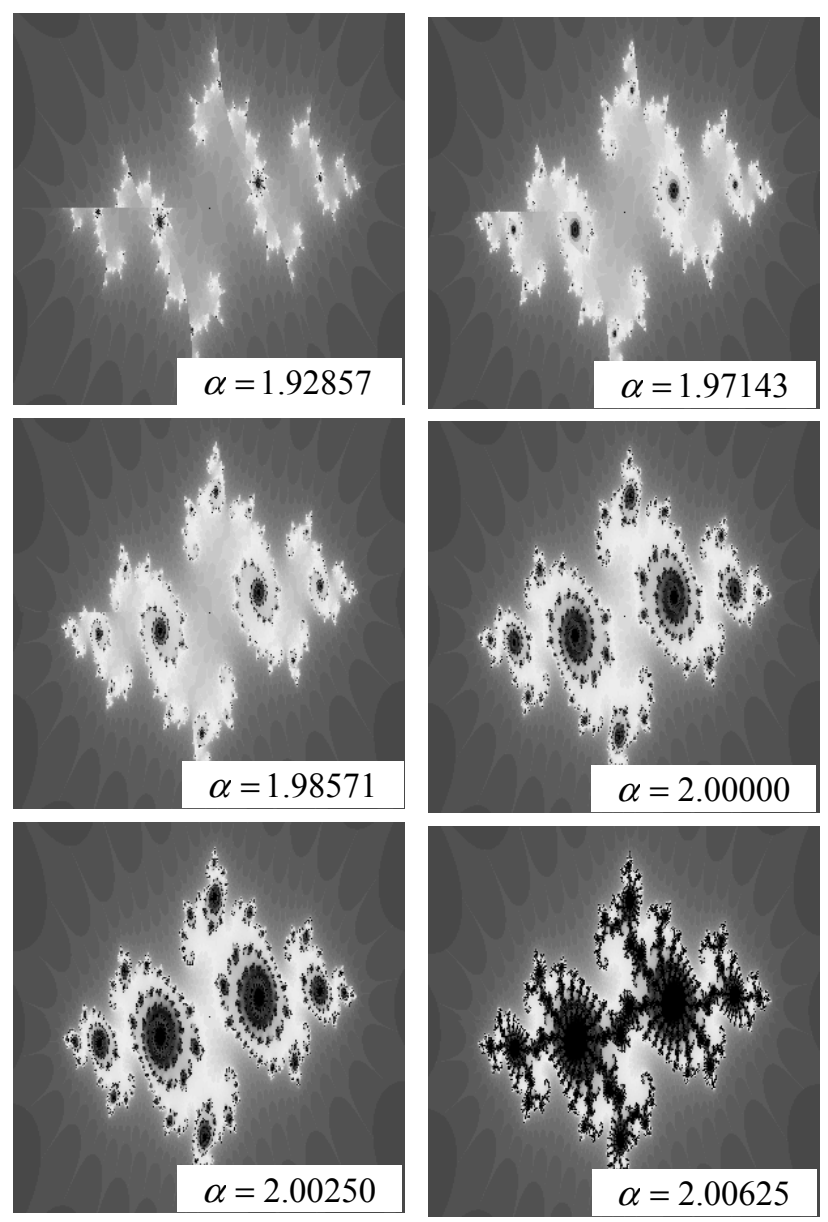

Fig.4 - Samples of Julia sets made by the students.

environment and equipment, it took average 15 seconds to make a fractal computer graphics in the $300 \times 300$ size. If a 15 seconds worth of a movie with 10 frames/sec is made, 150 graphics are needed. It takes 2250 seconds (37.5 minutes) in total. However, if we let 150 students make each figure, it needs just 15 seconds. Therefore, the presented lecture style can provide possibility of new education and research. The lecture style can be applied to the project and problem which requires vast execution time if it is done by a sequential procedure, for example, searching problem for suitable initial condition and/or optimal solution, making various and huge data, and so on. In this point of view, we consider that a small-scale and distributed education system, for example our MXGraphics shown in this paper, will be highlighted to realize new-style lecture. If the lecturer makes such an education system and distributes it to the students, and he can let them

Table 2. - Results of the auestionnaire

\begin{tabular}{l|lllll}
\hline & 5 & 4 & 3 & 2 & 1 \\
\hline Item 1 & $23 \%$ & $35 \%$ & $28 \%$ & $11 \%$ & $3 \%$ \\
Item 2 & $15 \%$ & $41 \%$ & $26 \%$ & $12 \%$ & $6 \%$ \\
Item 3 & $10 \%$ & $42 \%$ & $34 \%$ & $10 \%$ & $4 \%$ \\
\hline
\end{tabular}

execute the procedure in parallel, and can expect to the aforementioned feature, clarity of the purpose, students' sensitivity and responsibility of the result, and feedback on the their results. Thus, to support the lecture, the distributed education material system is important, and the project-style lecture with the system can be considered as one of data making and acquisition system.

\section{EVALUATION}

To evaluate the presented education material system and the project-style lecture, we asked students to fill out questionnaires, and tallied it up. The items of the questionnaires are as follows:

Questionnaire:

1. Could you be interested in the lecture?

2. Could you be satisfied in the lecture?

3. Could you understand the contents of the lecture?

4. What do you want to improve on the lecture?

We asked the students to answer the item 1, 2 and 3 of the questionnaire at five levels, that is, 1. Not, 2. Not much 3. Maybe, 4. Some and 5. Enough.

The result of tally up of questionnaire is also shown in Table 2. From the result, it is found that they were motivated and interested enough throughout the lecture. The educational effect was surely improved. However, a part of good achievement students had the opinions that the progress of the lecture should be a little speedy. It is a good opinion, and we consider that the former part of the lecture is a little dull, and it is one of our future works. Hereinbefore we can conclude that the presented educational material system and project-style lecture is effective.

\section{FUTURE AND ON-GOING WORKS}

We are developing a new education system, called "MXSounds", as a teaching material using sounds, and try to introduce the system to the lecture on the signal processing. Sound is a good material for teaching the important notion of the analysis and filtering of signals in the time or frequency domain.

This system runs on Windows ${ }^{\circledR}$ with the MaTX as same as the MaTXGraphics. We are now groping for the good project on the sound with the education system.

To give a supplementary information on the 
MaTX, MXGraphics and MXSounds, we describe the URL concerning each tools:

MaTX: http://www.matx.org

MXGraph,MXSounds:

http://furutalab.k.dendai.ac.jp

or http://www.hatalab.k.dendai.ac.jp

\section{CONCLUSIONS}

An education material system and a project-style lecture were presented in this paper in order to raise the level of low-achievement students and to interest other students simultaneously. We showed that such the lecture style can provide and improve clarity of the purpose, students' sensitivity and responsibility of the result, and feedback on the their results. Also, we discussed the possibility of the education material system, for example, as a distributed data-making/acquisition system. From the result of the questionnaire, it was shown that the presented lecture and education systems are effective.

\section{ACKNOWLEDGEMENT}

We appreciate that Prof. M. Koga, who developed MaTX software, gave us some valuable comments and suggestions.

\section{REFERENCES}

[1]Benoit Mandelbrot. Fractals and the measurement of roughness, the $19^{\text {th }}$ Japan Prize Laureate Lecture, Tokyo, Japan, 2002.

[2]Robert Walker, Charles Gregory Jr., Sunil Shah. MATRIXx: A Data Analysis, System Identification, Control Design and Simulation Package, Control Systems Magazine (1982). pp. 30-37.

[3]Inc. The Math Works, MATLAB User's Guide, 24 Prime Park Way, Natick, Mass. 01760-1500, USA, 1992.

[4]Masanobu Koga. MaTX/RtMaTX: A Freeware for Integrated CACSD. Proc. of CACSD'99, Kohala Coast-Island, Hawai, U.S.A. 1999, pp.451-456.

[5]S. Hatakeyama, Y. Pan. MaTX Aided Control Education, IEEE CCA (1999). pp.480-485.

[6]Tasuku Hoshino, Katsuhisa Furuta. Modeling and Simulation of Mechanical Systems Combination of a Symbolic Computation Tool and MaTX. Proc. of CACSD'99, Kohala Coast-Island, Hawai, U.S.A. 1999. pp. 462-467.

[7]J.J. Dongarra, C.B. Moler, J.R. Bunch, G.W. Stewart. LINPACK - User's guide, SIAM, Philadelphia, 1979.

[8]B.S. Garbow, J.M. Boyle, J.J. Dongarra, C.B. Moler. Matrix Eigen-system Routines - EISPACK Guide Extension. Springer-Verlag, 1977.

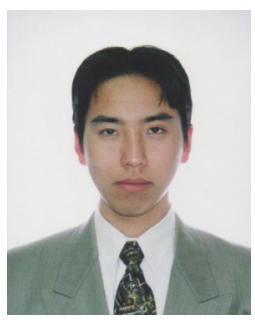

Masami Iwase received his Master's and Ph.D. degrees in Control Engineering from the Tokyo Institute of Technology in 1999 and 2001, respectively. He is currently a research assistant at the Tokyo Denki University since 2001. His main research areas are control theory and system identification based on the computation viewpoint. $\mathrm{He}$ is also engaged in education for the information and system engineering. $H e$ is a member of SICE and IEEE.

\section{Shoshiro Hatakeyama} received the Ph.D. degree in Engineering from the Tokyo Institute of Technology in 1980. From 1984, he was a research associate in the medical school of the UCSD, USA. He joined the Tokyo Denki University as a

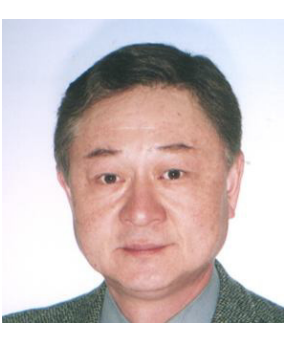
full-time lecturer from 1985, an associate professor from 1986, and a professor since 1993. He has interests in system engineering, system identification and chaos system. He is a member of SICE and IEEE.

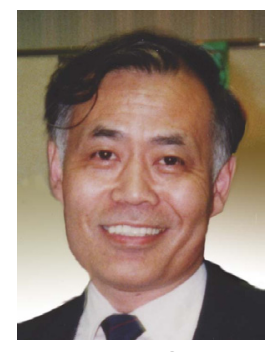

Katsuhisa Furuta received his B.S. M.S., and Ph.D.degrees in Engineering from Tokyo Institute of Technology in 1962, 1964, and 1967, respectively. He had been a Professor of Control Engineering, Tokyo Institute of Technology till the end of March in 2000. He was a Russell Severance Springer Visiting Professor, University of California at Berkeley in Match, 1997. He is currently Professor at Tokyo Denki University, Department of Computers and Systems Engineering. He was a Member of Science Council of Japan from 1997 to July, 2003. From 1994 to 1999 he was a Council Member of IFAC and the editor of Automatica in application from 1996 to 1999. He is now serving the Research Supervisor of Mine Detection and Clearance Activities, Japan Science and Technology, Co. from 2002. He also received an honorary doctorate from Helsinki University of Technology in 1998, and international awards including of IEEE CSS Distinguished Member in 1998, IEEE Third Millennium Medal in 2000. He is a Fellow of SICE, IEEE and IEE. 